\title{
Clima familiar y rendimiento académico en estudiantes universitarios durante la educación virtual en tiempos de Covid-19
}

\author{
Family climate and academic performance in university students during virtual education in times of Covid-19
}

Clima familiar e desempenho acadêmico em estudantes universitários durante a educação virtual em tempos de Covid-19

Anibal Sucari León

asucari@unap.edu.pe https://orcid.org/0000-0003-3746-9761

Universidad Nacional del Altiplano, Puno, Perú

\author{
Amilcar Giovanny Terán Dianderas \\ agteran@unap.edu.pe \\ https://orcid.org/0000-0002-8669-1353
}

Universidad Nacional del Altiplano, Puno, Perú
Haydee Clady Ticona Arapa

hcticona@unap.edu.pe

https://orcid.org/0000-0003-3963-9218

Universidad Nacional del Altiplano, Puno, Perú

Nancy Chambi Condori

nchambi@unap.edu.pe

https://orcid.org/0000-0002-4520-4707

Universidad Nacional del Altiplano, Puno, Perú

Artículo recibido en junio 2021, revisado en julio 2021, arbitrado en agosto 2021 y publicado en septiembre 2021

\section{RESUMEN}

El estudio tuvo como objetivo demostrar la influencia del clima familiar en el rendimiento académico de los estudiantes universitarios del I al V ciclo de la Escuela Profesional de Ingeniería de Minas y Educación Inicial de la Universidad Nacional del Altiplano, durante la educación virtual en tiempos de Covid-19. La investigación es de tipo básica con nivel descriptivo y diseño no experimental de corte transversal en el cual se empleó el método científico y estadístico, se trabajó con una muestra de 200 estudiantes, 70 mujeres y 130 varones, con muestreo probabilístico estratificado en una población de 416 estudiantes. Logrando como resultado que si existe una relación positiva fuerte $y$ estadísticamente significativa entre el clima familiar y el rendimiento académico con una significancia del 5\% en los estudiantes de Ingeniería de Minas y Educación inicial. Concluyendo que es importante tener un buen clima familiar para lograr un rendimiento académico positivo.

Palabras clave: Clima familiar; Dimensiones; Estudiantes universitarios; Nivel de logro; Rendimiento académico

\section{ABSTRACT}

The objective of the research was to demonstrate the influence of the family climate on the academic performance of university students from I to $\mathrm{V}$ cycle of the Professional School of Mining Engineering and Initial Education of the Universidad Nacional del Altiplano, during the virtual education in times of Covid-19. The research is of basic type with descriptive level and nonexperimental design of transversal cut in which the scientific and statistical method was used, working with a sample of 200 students, 70 women and 130 men, with stratified probabilistic sampling in a population of 416 students. As a result, there is a strong and statistically significant positive relationship between family climate and academic performance with a significance of $5 \%$ in students of Mining Engineering and Initial Education. Concluding that it is important to have a good family climate to achieve a positive academic performance.

Key words: Family climate; Dimensions; College students; Achievement level; Academic performance

\section{RESUMO}

O objetivo da pesquisa foi demonstrar a influência do clima familiar no desempenho acadêmico dos estudantes universitários do I ao $\mathrm{V}$ ciclo da Escola Profissional de Engenharia de Minas e Educação Inicial da Universidade Nacional do Altiplano, durante a educação virtual nos tempos do Covid-19. A pesquisa é de um tipo básico com um nível descritivo e desenho transversal não experimental no qual foi utilizado o método científico e estatístico, trabalhando com uma amostra de 200 estudantes, 70 mulheres e 130 homens, com amostragem probabilística estratificada em uma população de 416 estudantes. Como resultado, existe uma forte e estatisticamente significativa relação positiva entre o clima familiar e o desempenho acadêmico, com um significado de 5\% nos estudantes de Engenharia de Minas e Educação Inicial. Concluímos que é importante ter um bom clima familiar a fim de alcançar um desempenho acadêmico positivo.

Palavras-chave: Clima familiar; Dimensões; Estudantes universitários; Nível de realização; desempenho acadêmico 


\section{INTRODUCCIÓN}

El rendimiento académico es un constructo susceptible de adoptar valores cuantitativos y cualitativos, a través de los cuales existe una aproximación a la evidencia y dimensión del perfil de habilidades, conocimientos, actitudes y valores desarrollados por el alumno en el proceso de enseñanza aprendizaje señaló el experto Navarro (2005). Por otra parte, en el rendimiento académico de los estudiantes intervienen múltiples causas, desde variables que tienen que ver con los determinantes personales hasta factores asociados a aspectos sociales afirmó Barahona (2014).

Adicionalmente, en el mismo intervienen diferentes variables externas al curso, como la calidad de los conocimientos del docente virtual, el programa educativo, la planeación previa del curso, la actitud hacia la asignatura, la personalidad del estudiante y la motivación hacia el seminario por otra parte menciona que los docentes universitarios se deben capacitarse en los diferentes proceso de enseñanza en ambientes virtuales ya que el docente virtual es un orientador y el estudiante es quién de manera autónomo debe liderar el proceso de su aprendizaje destacaron González-Guerrero et al. (2010).

Los enfoques de aprendizaje y el rendimiento de aprendizaje no tienen relación en la educación virtual, ya que tuvieron la forma de evaluar mediante el control de actividades programadas durante el curso consideraron (Recio-Saucedo, 2005). Como lo señalaron Borgobello y Roselli (2016) que existe diferencia significativa entre las calificaciones y el grado de utilización de las plataformas virtuales.

En el adecuado rendimiento académico inciden diversos factores y no puede explicarse solo por la
Institución educativa, aunque este sí es uno de los factores a tener en cuenta. Existen otros, como la inteligencia cognitiva tradicional y el rendimiento académico previo, entre otras variables declararon Páez-Cala y Castaño-Castrillón (2019).

La organización familiar, referida al grado de planificación y el establecimiento de funciones y normas dentro de una familia, incide decisivamente en la adaptación de los hijos, pues viven en una familia de alta organización tienen mayores niveles de adaptación general, emocional, familiar social y una mejor salud consideraron Amezcua-Membrilla et al. (2002).

El entorno familiar ocupa un lugar importante, el mismo es un conjunto de interacciones propias de la convivencia familiar, que afectan el desarrollo del individuo, manifestándose también en la vida académica. La influencia del padre y la madre, o del adulto responsable del estudiante, influye significativamente en la vida académica. Un ambiente familiar propicio, marcado por el compromiso, incide en un adecuado desempeño académico, así como una convivencia familiar democrática entre padres e hijos. Se asocia la convivencia familiar democrática con un mejor desempeño académico, que se plasma en variables como motivación, percepción de competencia y atribución de éxito académico; no sucede lo mismo en estudiantes marcados por ambientes familiares autoritarios e indiferentes, de parte de sus padres revelaron (Garbanzo-Vargas, 2012). La importancia del papel que juega el clima familiar en las facilidades u obstáculos de adaptación que tendrá el adolescente en su entorno social plantearon (Verdugo-Lucero et al., 2014). 
Las relaciones entre los miembros de la familia constituyen un ambiente decisivo para el desarrollo y configuración del autoconcepto en la adolescencia, por otra parte, indica en relación al clima familiar son los factores de cohesión, expresividad y organización familiar los que guardan una relación positiva con el autoconcepto en todas sus áreas definieron (Mestre Escriva et al., 2001). Así mismo ratificaron (Torres-Velázquez y Rodríguez-Soriano, 2006) que el rendimiento escolar también depende del contexto en el que se desarrolle la familia y el estudiante, porque es importante la percepción que los jóvenes tengan acerca de la valoración positiva o negativa de su familia hacia ellos, su percepción del apoyo que aquélla les presta, la percepción de los padres de las tareas, sus expectativas futuras, su comunicación con los estudiantes y su preocupación por ellos, así mismo manifiestan que en las universidades por medio de la tutoría se pueda apoyar en su rendimiento académico del estudiante.

Por otra parte, el entorno urbano o rural sí influye de manera diferente en las variables del clima familiar percibidas por los adolescentes mencionaron (Jiménez-Tallón et al., 1999). La integración en el grupo no ejerció un efecto determinante sobre la satisfacción familiar y sí lo hicieron la autoestima y la autoeficacia, puesto que éste no sólo depende de la actuación de los padres sino también de las influencias que reciben sus hijos de otros contextos manifestaron Teresa-Vega y De Dios-De Dios (2006).

Se suma lo que Trevor-Mthimunye y MeganDaniels (2019) revelan de la necesidad de que el estudiante esté preparado para el programa, los estudiantes tienen necesidades múltiples de apoyo de la familia y de la institución de enseñanza superior. Si bien es cierto que es deber de todos los responsables de la educación dedicar tiempo y esfuerzo suficiente para que los alumnos y alumnas de este nivel educativo empiecen a convertirse en hábiles estudiantes, antes de que su rendimiento potencial se vea mermado por el desconocimiento o la falta de aplicación de dichas estrategias y motivaciones consideraron (Maquillón-Sánchez y Hernández-Pina, 2011).

Por todo lo mencionado, en la presente investigación se propuso como objetivo demostrar la influencia del clima familiar en el rendimiento académico de los estudiantes universitarios del I al V ciclo de la Escuela Profesional de Ingeniería de Minas y Educación Inicial de la Universidad Nacional del Altiplano, matriculados en el semestre 2020-I durante la educación virtual en tiempos de Covid-19, la cual está conformada por estudiantes mujeres en Educación Inicial y estudiantes varones en Ingeniería de Minas, también se pretende conocer el nivel del clima familiar de los estudiantes y el grupo que tiene mejor rendimiento académico todos ellos pertenecientes a la Universidad Nacional del Altiplano de Puno, donde se está aplicando la educación virtual a consecuencia de la pandemia mundial del COVID-19.

Este trabajo de investigación se realizó porque los estudiantes de la Universidad Nacional del Altiplano están en una educación virtual a causa de la pandemia mundial Covid-19, estando permanente en una convivencia entre los estudiantes universitarios y los miembros de familia, cabe mencionar que en la educación presencial un gran porcentaje de estudiantes universitarios viven solos en la ciudad de Puno ya que provienen de diferentes provincias de Puno y regiones del Perú. 


\section{MÉTODO}

La investigación es de tipo cuantitativo de un nivel descriptivo correlacional transversal porque describe los hechos y fenómenos en un solo momento, siguiendo un diseño no experimental ya que no se manipularon ninguna de las variables.

El estudio se realizó con los estudiantes que se matricularon en el semestre 2020-I, para el recojo de los datos se aplicó el cuestionario en el semestre 2020-II, mes de diciembre del 2020.

Los materiales que se utilizaron fueron el reporte de coordinación académica de la escuela profesional de Ingeniería de Minas y Educación Inicial los estudiantes matriculados en el semestre 2020-I, se utilizó un cuestionario virtual en Google por la educación a distancia debido a la pandemia del COVID-19 dicho instrumento fue validado mediante juicio de expertos. Las variables que se analizaron en la investigación fueron el clima familiar y el rendimiento académico de los estudiantes de Ingeniería de Minas y Educación Inicial. Así mismo se realizó una prueba piloto de 20 estudiantes para encontrar el coeficiente de confiabilidad obteniendo un valor de Alfa de Cronbach de 0.71 y 0.83 para los instrumentos, deduciendo que el instrumento de investigación tiene una confiabilidad aceptable.

Respecto a las técnicas de procesamiento y análisis de datos se empleó tablas de distribución de frecuencia, en la cual se procesaron las respuestas del instrumento utilizado, logrando obtener el gráfico de barras mostrando su mejor representación de los hallazgos, al mismo tiempo se calculó el coeficiente de correlación de "r" de Pearson y su prueba de hipótesis para la significancia correspondiente.

La población de estudio estuvo conformada por 416 estudiantes pertenecientes a la Universidad Nacional del Altiplano todos matriculados en el semestre 2020-I se consideró desde I al V ciclo, la Escuela Profesional de Ingeniería de Minas con 271 estudiantes y la Escuela Profesional de Educación Inicial con 145 estudiantes. Se aplicó el muestreo probabilístico estratificado, obteniendo una muestra representativa de 200 estudiantes del total de la población, 130 estudiantes de Ingeniería de Minas y 70 estudiantes de Educación Inicial.

\section{RESULTADOS Y DISCUSIÓN}

El trabajo de investigación presenta en los resultados las mediciones y análisis de las variables de estudio; clima familiar y rendimiento académico de los estudiantes de Educación Inicial y estudiantes de Ingeniería de Minas de la Universidad Nacional del Altiplano de Puno que corresponden al semestre académico 2020-I.

En la Tabla 1 se muestra que la variable de clima familiar está compuesta por tres dimensiones, observando que la dimensión de relación familiar tiene mayor puntaje en promedio y sumatoria en puntaje, seguida de la dimensión de desarrollo familiar y estabilidad familiar, esto quiere decir que las estudiantes de Educación Inicial mantienen buena relación familiar. 
Tabla 1. Dimensiones del clima familiar en los estudiantes de Educación Inicial.

\begin{tabular}{lcccc}
\hline \multicolumn{1}{c}{$\begin{array}{c}\text { Estadígrafos } \\
\text { descriptivos }\end{array}$} & $\begin{array}{c}\text { Clima } \\
\text { Familiar }\end{array}$ & $\begin{array}{c}\text { Relación } \\
\text { Familiar }\end{array}$ & $\begin{array}{c}\text { Desarrollo } \\
\text { Familiar }\end{array}$ & $\begin{array}{c}\text { Estabilidad } \\
\text { Familiar }\end{array}$ \\
\hline Media & 50,10 & 18,69 & 16,66 & 14,76 \\
Mediana & 50,00 & 19,00 & 17,00 & 14,00 \\
Moda & 53 & 24 & $16 \mathrm{a}$ & 16 \\
Desviación Estándar & 10,232 & 3,828 & 3,683 & 4,573 \\
Varianza & 104,700 & 14,653 & 13,562 & 20,911 \\
Asimetría &, 179 &,- 286 &, 100 &, 482 \\
Curtosis &,- 576 &,- 694 &,- 656 &,- 698 \\
Rango & 45 & 14 & 14 & 17 \\
Mínimo & 28 & 10 & 10 & 8 \\
Máximo & 73 & 24 & 24 & 25 \\
Suma & 3507 & 1308 & 1166 & 1033 \\
\hline
\end{tabular}

En la Tabla 2 donde se expresa que la dimensión de relación familiar presenta mayor promedio y sumatoria en puntajes, seguida de la dimensión de desarrollo familiar y estabilidad familiar muy similar a la Tabla 1, esto quiere decir que los estudiantes de Ingeniería de Minas mantienen buena relación familiar.

Tabla 2. Dimensiones del clima familiar en los estudiantes de Ingeniería de Minas.

\begin{tabular}{lcccc}
\hline $\begin{array}{c}\text { Estadígrafos } \\
\text { descriptivos }\end{array}$ & $\begin{array}{c}\text { Clima } \\
\text { Familiar }\end{array}$ & $\begin{array}{c}\text { Relación } \\
\text { Familiar }\end{array}$ & $\begin{array}{c}\text { Desarrollo } \\
\text { Familiar }\end{array}$ & $\begin{array}{c}\text { Estabilidad } \\
\text { Familiar }\end{array}$ \\
\hline Media & 49,11 & 18,06 & 15,63 & 15,42 \\
Mediana & 50,00 & 18,00 & 16,00 & 16,00 \\
Moda & 50 & 24 & $15 a$ & 18 \\
Desviación Estándar & 13,535 & 4,811 & 4,457 & 5,995 \\
Varianza & 183,198 & 23,143 & 19,863 & 35,935 \\
Asimetría &,- 598 &,- 728 &,- 361 &,- 225 \\
Curtosis &, 331 &, 170 &, 188 &,- 839 \\
Rango & 67 & 21 & 22 & 24 \\
Mínimo & 7 & 3 & 2 & 2 \\
Máximo & 74 & 24 & 24 & 26 \\
Suma & 6384 & 2348 & 2032 & 2004 \\
\hline
\end{tabular}


La Figura 1 muestra una distribución sobre la variable rendimiento académico de las estudiantes de Educación Inicial, donde el promedio ponderado más alto es de 16 que corresponden a 20 estudiantes, seguida del promedio de 17 que corresponden a 17 estudiantes y el promedio de 15 que corresponde a 15 estudiantes, esto quiere decir que los estudiantes están en un nivel de logro previsto.

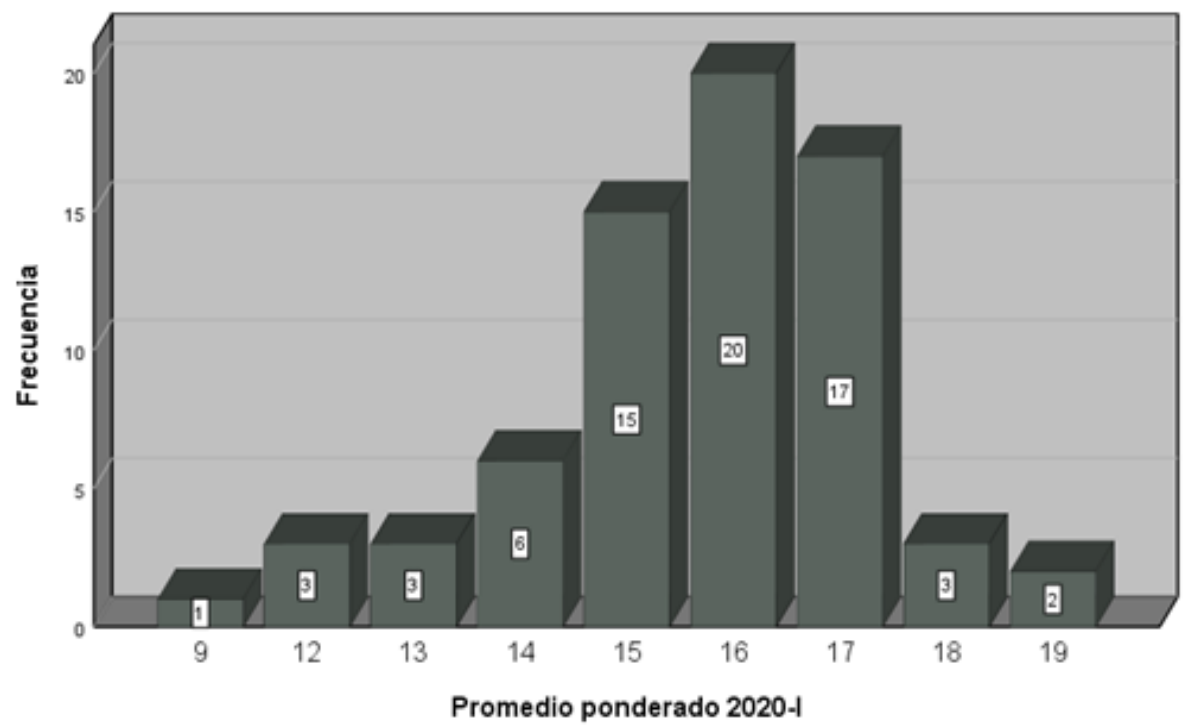

Figura 1. Rendimiento académico de estudiantes de Educación Inicial.

La Figura 2 muestra la distribución de la variable rendimiento académico de los estudiantes de Ingeniería de Minas, donde el promedio ponderado más alto es de 14 puntos que corresponden a 46 estudiantes, seguida del promedio 13 puntos que corresponden a 28 estudiantes y el promedio de 12 puntos que corresponden a 20 estudiantes, esto quiere decir que los estudiantes están entre el nivel de logro en proceso a previsto.

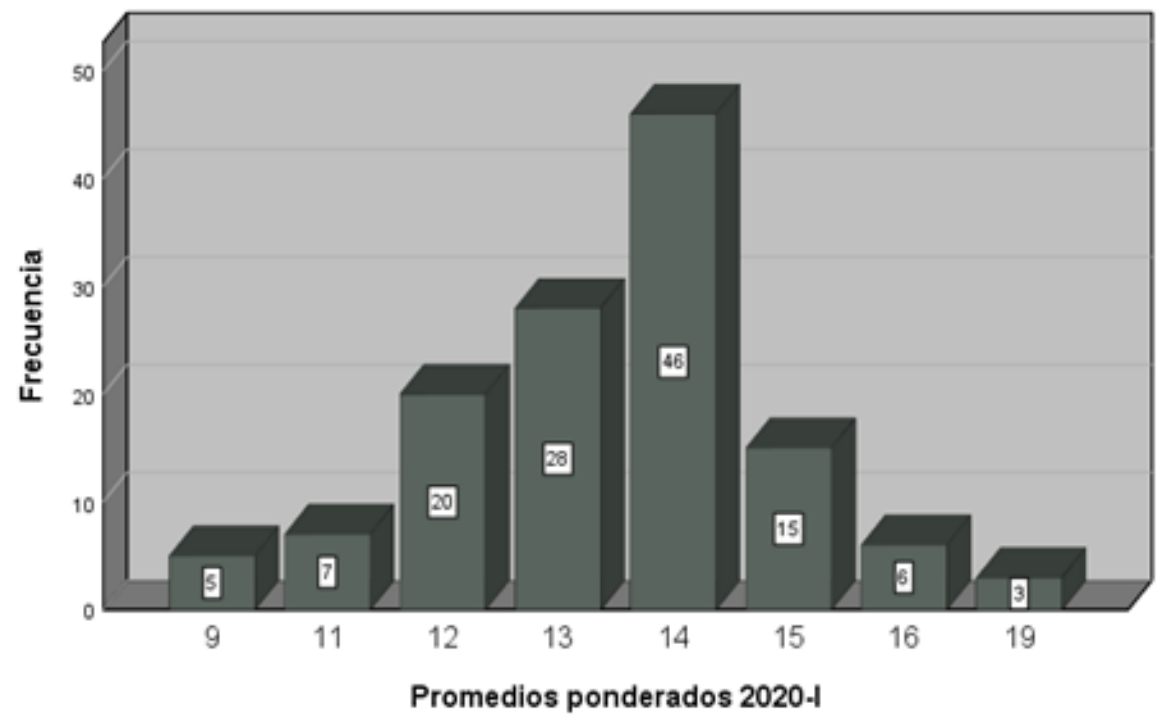

Figura 2. Rendimiento académico de estudiantes de Ingeniería de Minas. 
La Figura 3 muestra la distribución de la variable clima familiar medido en las estudiantes de Educación Inicial donde resalta que 38 estudiantes mantienen buen clima familiar esto es más del 50\% de estudiantes, seguidamente del clima familiar regular con 25 estudiantes.
La Figura 3 muestra la distribución de la variable clima familiar medido en las estudiantes de Educación Inicial donde resalta que 38 estudiantes mantienen buen clima familiar esto es más del 50\% de estudiantes, seguidamente del clima familiar regular con 25 estudiantes.

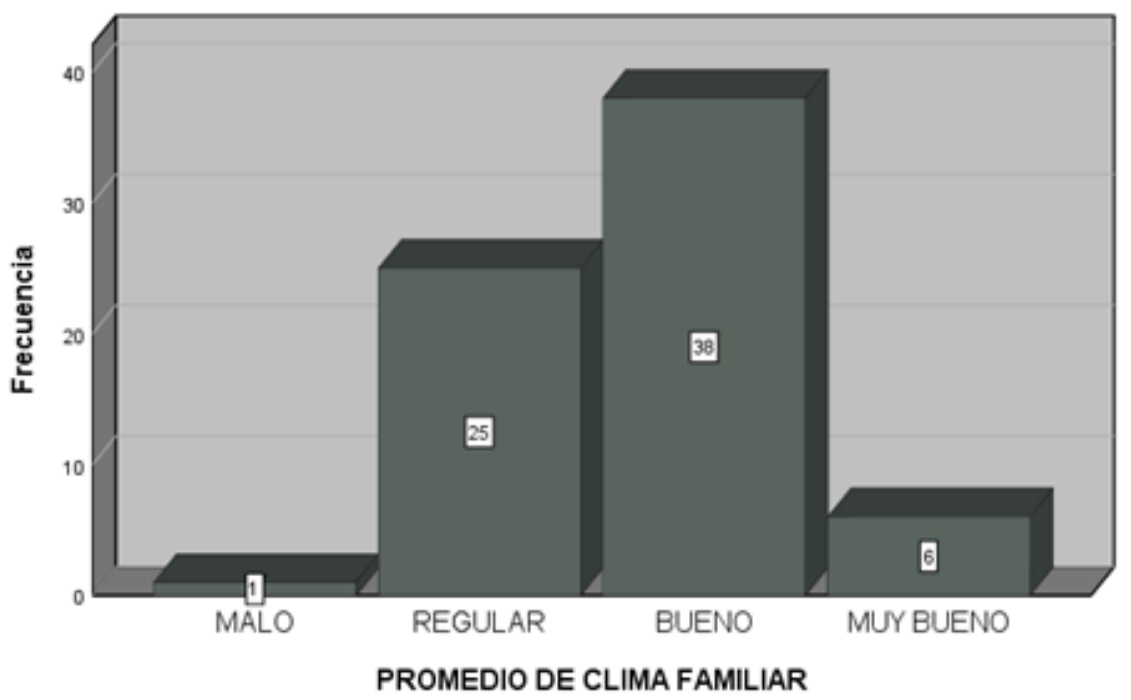

Figura 3. Nivel de clima familiar en estudiantes de Educación Inicial.

La Figura 4 muestra la distribución de la variable clima familiar medido en los estudiantes de Ingeniería de Minas donde la mayor cantidad de estudiantes tiene un buen clima familiar que corresponde a 64 estudiantes seguidos de regular con 43 estudiantes, muy similar a la Figura 3.

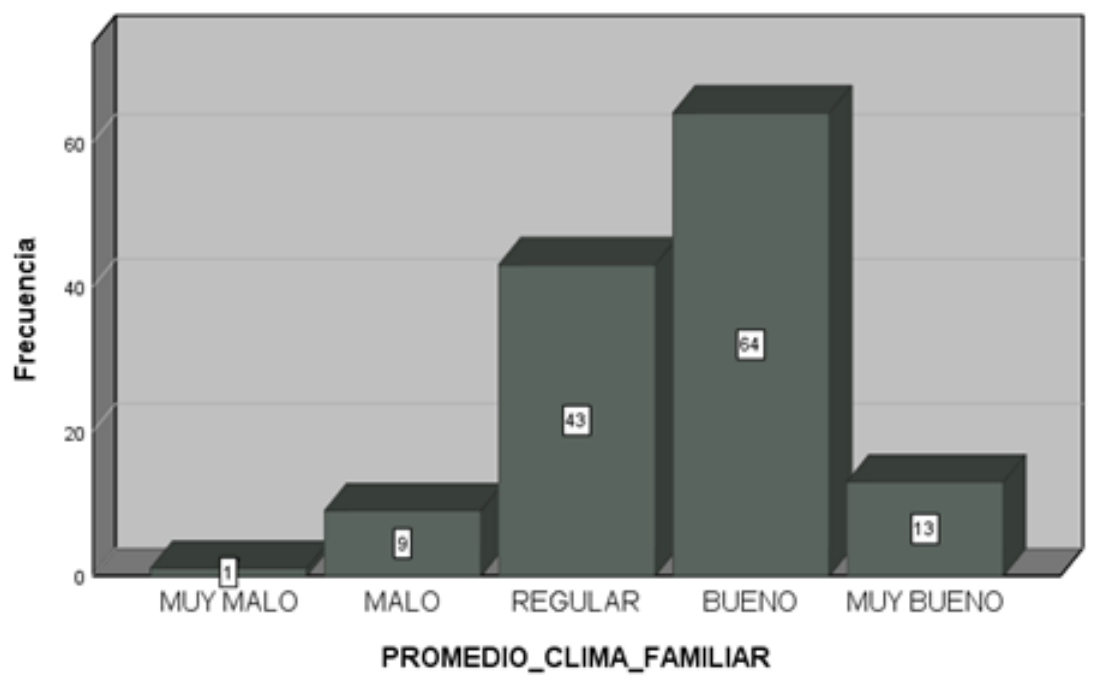

Figura 4. Nivel de clima familiar en estudiantes de Ingeniería de Minas. 
Respecto al contrastes de la hipótesis se empleó el software SPSS v.25 en el cual nos permite optimizar los diferentes procedimientos que corresponde a los cálculos estadísticos, ya sea para los estudiantes de Educación Inicial y Estudiantes de Ingeniería de Minas, mostrando como resultado en las siguientes tablas.

En la Tabla 3 se presentan los resultados de la prueba de hipótesis, donde se observa que existe una relación positiva $(\mathrm{r}=0,770)$ y estadísticamente muy significativa $\left({ }^{*}\right)$ entre las variables de estudio clima familiar y rendimiento académico en las estudiantes de Educación Inicial, esto quiere decir que mientras se mantenga un mejor clima familiar como resultado se tendrá un mejor rendimiento académico.

Tabla 3. Coeficiente de correlación entre clima familiar y rendimiento académico.

\begin{tabular}{llcc}
\hline & & $\begin{array}{c}\text { Rendimiento académico en } \\
\text { Educación Inicial }\end{array}$ & $\begin{array}{c}\text { Rendimiento académico en } \\
\text { Ingeniería de Minas }\end{array}$ \\
\hline Clima familiar & Correlación de Pearson & $0,770^{* *}$ & $0,726^{* *}$ \\
& Sig. (bilateral) & 0,000 & 0,000 \\
& $\mathrm{~N}$ & 70 & 130
\end{tabular}

Por otra parte, la Tabla 3 presenta los resultados de la prueba de hipótesis, donde se observa que si existe una relación positiva $(r=0,726)$ y estadísticamente muy significativa ${ }^{* *}$ ) entre las variables de estudio clima familiar y rendimiento académico en los estudiantes de Ingeniería de Minas, esto quiere decir que si se mantiene un buen clima familiar es muy favorable para lograr un mejor rendimiento académico en los estudiantes o de lo contrario si se presenta el peor clima familiar tendremos un rendimiento académico bajo.

En la tabla 4 donde se presenta los coeficientes de correlación de las dimensiones del clima familiar frente al rendimiento académico de las estudiantes de
Educación Inicial, donde se observa que si existe una relación positiva en las tres dimensiones, relación familiar $(r=0,648)$, desarrollo familiar $(r=0,663)$ y estabilidad familiar $(r=0,684)$, del mismo modo los coeficientes de correlación de las dimensiones del clima familiar frente al rendimiento académico de los estudiantes de Ingeniería de Minas, se evidencia una relación positiva en las tres dimensiones, relación familiar $(r=0,684)$, desarrollo familiar $(r=0,654)$ $y$ estabilidad familiar $(r=0,669)$, en ambos casos estadísticamente son muy significativas ${ }^{* *}$ ) en las tres dimensiones del clima familiar, estos resultados corroboran la contrastación de la hipótesis general. 
Tabla 4. Coeficiente de correlación entre las dimensiones y rendimiento académico

\begin{tabular}{llcc}
\hline & & $\begin{array}{c}\text { Rendimiento académico en } \\
\text { Educación Inicial }\end{array}$ & $\begin{array}{c}\text { Rendimiento académico en } \\
\text { Ingeniería de Minas }\end{array}$ \\
\hline Dimensión Relación Familiar & Correlación de Pearson & $0,648^{* *}$ & $0,684^{* *}$ \\
& Sig. (bilateral) & 0,000 & 0,000 \\
& $\mathrm{~N}$ & 70 & 130 \\
Dimensión Desarrollo Familiar & Correlación de Pearson & $0,663^{* *}$ & $0,654^{* *}$ \\
& Sig. (bilateral) & 0,000 & 0,000 \\
& $\mathrm{~N}$ & 70 & 130 \\
Dimensión Estabilidad Familiar & Correlación de Pearson & $0,684^{* *}$ & $0,669^{* *}$ \\
& Sig. (bilateral) & 0,000 & 0,000 \\
& $\mathrm{~N}$ & 70 & 130 \\
\hline
\end{tabular}

\section{Discusión}

Según a los resultados existe una relación directa y significativa entre el clima familiar y el rendimiento académico en estudiantes de Educación Inicial e Ingeniería de Minas de la Universidad Nacional del Altiplano de Puno. Dichos resultados son ratificados por (Patiño-Rosales, 2019) donde encontraron que si existe una relación significativa entre el clima familiar con el rendimiento académico de los estudiantes de la escuela profesional de Odontología de la Universidad Peruana los Andes Huancayo - 2018. Así mismo, AroquipaDurán y Sucari-León (2019) concluyeron que si existe una relación fuerte entre el clima familiar y el rendimiento académico, estadísticamente muy significativa en los estudiantes del centro de idiomas de la Universidad Nacional José María Arguedas de Andahuaylas 2018-I. Como señalaron (Antezana-Morón, 2015) que el clima familiar y el rendimiento académico de los estudiantes de la Universidad Nacional de Barranca, Lima, 2014, se relacionan significativamente, porque los datos de la estadística descriptiva muestran porcentajes altos y la estadística inferencial, mediante el coeficiente $r$ de Pearson $(r=0.875)$, confirma la correlación.

Los resultados muestran que las estudiantes mujeres de Educación Inicial tienen un rendimiento académico promedio mayor encontrándose en el nivel de logro previsto y los estudiantes varones de Ingeniería de Minas tienen un rendimiento académico promedio menor encontrándose en un nivel de logro en proceso todos ellos pertenecientes del I ciclo al $\mathrm{V}$ ciclo, este nivel de logro bajo se puede apoyar en Jara et al. (2013) donde mencionaron que los estudiantes de los primeros años carecen de hábitos y estrategias de estudio, ya que proceden la mayoría de colegios estatales. Pero Zimmer-Ferreira et al. (2020) demostraron en su investigación una relación multifacética entre los adolescentes e Internet. Pero Qutishat et al., (2020) afirmaron que la prevalencia de la nomofobia entre los estudiantes universitarios fue alta, del 99,33\%, 
mostraron evidencia de una asociación con el bajo rendimiento académico.

En términos generales, los estudiantes muestran promedios aceptables, la mayoría cuenta con una familia nuclear (papá, mamá e hijos) y viven con ellos, por tanto, se puede concluir que cuentan con el apoyo de ambos padres y con las condiciones favorables y recursos económicos suficientes para culminar sus estudios enfatizaron Sotelo-Castillo et al. (2011). Por su parte, Bocanegra-Pérez (2017) manifiesta que no existen diferencia significativa entre las dimensiones de relaciones, desarrollo y estabilidad según género, poca varones y mujeres perciben que las interacciones en sus familias en las diversas dimensiones del clima social familiar no presentan variaciones. Vedaa et al. (2019) sostuvieron que el trastorno del insomnio tiene consecuencias notables en aspectos de capacidad de aprendizaje y éxito académico en la educación superior.

El clima social familiar en general se encuentra en una categoría media debido a que las dimensiones de relación, desarrollo y estabilidad obtuvieron $45 \%, 59 \%$ y $68 \%$ respectiva mente ello quiere decir que las relaciones saludables en el hogar están ausentes en el clima social familiar lo que resulta un clima no apropiado para la familia argumentaron Sandoval-Oviedo (2017). Los residentes evaluados, afirma que las largas jornadas de trabajo y los turnos limitan las actividades académicas y, el 91\% de ellos, considera que los exámenes no evalúan realmente lo aprendido durante la residencia concluyeron González-Mariño (2019).

Patiño-Rosales (2019) afirmaron que sí existe una relación significativa entre el clima familiar con el rendimiento académico de los estudiantes de la escuela profesional de Odontología de la Universidad Peruana los Andes Huancayo - 2018, por otra parte, no se encontró la relación significativa del rendimiento académico con las dimensiones de desarrollo y estabilidad familiar, pero si existe una relación significativa entre la dimensión de relación familiar con el rendimiento académico.

Por su parte Aroquipa-Durán y Sucari-León, 2019 concluyeron en su trabajo de investigación que, si existe una relación fuerte entre el clima familiar y el rendimiento académico, estadísticamente muy significativa en los estudiantes del centro de idiomas de la Universidad Nacional José María Arguedas de Andahuaylas 2018-I, así mismo tiene relación el rendimiento académico con cada dimensión del clima familiar. Así mismo Díaz- y Yaringaño-L. (2014) afirmaron que existe relación significativa entre clima familiar y el afrontamiento positivo; se observa una relación significativa entre las dimensiones: Relaciones, Desarrollo y Estabilidad del clima social familiar con el Afrontamiento Positivo al estrés.

El clima familiar y el rendimiento académico de los estudiantes de la Universidad Nacional de Barranca, Lima, 2014, se relacionan significativamente, porque los datos de la estadística descriptiva muestran porcentajes altos y la estadística inferencial, mediante el coeficiente $\mathrm{r}$ de Pearson $(\mathrm{r}=0.875)$, confirma la correlación, señalaron Antezana-Morón (2015). Como lo describieron Gómez-Sánchez et al. (2011) que existe correlación directa y moderada entre la satisfacción con la carrera elegida y el rendimiento académico percibido $(r=0.444$, sig. $=0.000)$; por su parte, también hay una correlación moderada como evidencia de la asociación entre la variable 
promedio y el rendimiento académico percibido $(\mathrm{r}$ $=0.525$, sig. $=0.000)$.

Zimmer-Ferreira et al. (2020) demostraron en su investigación una relación multifacética entre los adolescentes e Internet. La red implica un intrincado tejido de interacciones, lo que lleva a diversos comportamientos y actitudes que reflejan que se reflejan en la salud del adolescente. Pero Qutishat et al. (2020) afirmaron que la prevalencia de la nomofobia entre los estudiantes universitarios fue alta, del 99,33\%, y especialmente entre las mujeres y los resultados mostraron evidencia de una asociación entre el bajo rendimiento académico y la nomofobia severa, que no fue estadísticamente significativa.

Edel Navarro (1997) analiza los resultados de su investigación y concluye que los alumnos muestran un desarrollo satisfactorio de sus habilidades verbales y matemáticas al ingreso a la preparatoria. Por otra parte Jara et al. (2013) afinaron que la mayoría de los estudiantes de medicina del primer año de estudios participantes del presente estudio proceden de colegios estatales, carecen de hábitos y estrategias de estudio, tienen autoestima moderada a baja, su nivel socioeconómico es bajo, residen en zonas urbano marginales sin vivienda propia $\mathrm{y}$ algunos de ellos tienen afecciones físicas que merman su salud; todo lo anterior podría influir en el rendimiento académico que presentan. Pero Cahuana-Cuentas et al. (2019) mencionaron que la familia y variables sociolaborales tienen un impacto significativo en los niveles de residencia de las personas con discapacidad física y sensorial, además de contar con apoyo económico y trabajo propio son factores predictores de niveles de resiliencia más altos.
Actualmente, el e-learning se perfila como solución a los problemas a los que la enseñanza tradicional no puede dar respuesta, sin embargo, por sí sólo no garantiza una educación de mayor calidad ni un mayor rendimiento en el aprendizaje. Por otra parte, existen numerosas investigaciones que demuestran que los estudiantes aprenden con más efectividad cuando se les enseña con sus estilos de aprendizaje predominantes, pero también se constata que es difícil llevar a la práctica en una clase tradicional la adaptación de la docencia a los Estilos de Aprendizaje de los alumnos consideraron Gallego-Rodríguez y Martinez-Caro, (2003).

\section{CONCLUSIONES}

Existe una relación positiva y estadísticamente muy significativa entre el clima familiar $y$ rendimiento académico ya sea en los estudiantes de Educación inicial e Ingeniería de Minas de la Universidad Nacional del Altiplano de Puno, matriculados en el semestre 2020-I, esto nos permite afirmar que es muy positivo tener el mejor clima familiar para así lograr el mejor rendimiento académico ya sea en mujeres y varones, de lo contrario se tendrá un rendimiento muy bajo.

Existe una relación positiva y estadísticamente significativa entre las dimensiones del clima familiar y el rendimiento académico de los estudiantes de Educación Inicial e Ingeniería de Minas de la Universidad Nacional del de Puno, matriculados en el semestre 2020-I, observándose que existe la relación fuerte entre las dimensiones relación familiar, desarrollo familiar y estabilidad familiar con el rendimiento académico de los estudiantes.

Respecto al rendimiento académico se pudo observar que las estudiantes de Educación Inicial 
se encuentran en un nivel de logro previsto, con los promedios más altos que varían desde 15 a 17 la mayor cantidad de estudiantes y los estudiantes de ingeniería de Minas se encuentran en un nivel de logro en proceso a previsto con los promedios más altos que varían entre 12 a 14, esto nos permite afirmar que las mujeres presentan un mejor rendimiento académico en la Universidad Nacional del Altiplano de Puno.

\section{REFERENCIAS}

Amezcua-Membrilla, J. A., Pichardo-Martínez, C., y Fernández de Haro, E. (2002). Importancia del clima social familiar en la adaptación personal y social de los adolescentes. Revista de Psicología General y Aplicada: Revista de La Federación Española de Asociaciones de Psicología, 55(4), 575-590. https://dialnet.unirioja.es/servlet/ articulo? codigo $=294345$

Antezana-Morón, E. del R. (2015). El clima familiar y su relación con el rendimiento académico de los estudiantes de la Universidad Nacional de Barranca, Lima 2014 [Universidad Nacional de Educación Enrique Guzmán y Valle]. In 2017. http://repositorio.une.edu.pe/ bitstream/handle/UNE/1899/T025_46253683T. pdf? sequence $=1$ \&is Allowed $=y \% 0$ Ahttp:// repositorio.une.edu.pe/bitstream/handle/ UNE/964/TM AD-Ad L319 2014. pdf? sequence $=1$ \&isAllowed $=y$

Aroquipa-Durán, Y., y Sucari-León, R. (2019). Clima familiar y rendimiento académico en estudiantes del Centro de Idiomas de la Universidad Nacional José María Arguedas -Andahuaylas2018. Puriq, 1(1), 12

Barahona-U, P. (2014). Factores determinantes del rendimiento académico de los estudiantes de la Universidad de Atacama. Estudios Pedagógicos (Valdivia), 40(1),25-39.https://doi.org/10.4067/ s0718-07052014000100002

Bocanegra-Pérez, A. (2017). Inteligencia emocional, clima social familiar y rendimiento académico en estudiantes del quinto de secundaria de las
Instituciones educativas del distrito de Comas [Universidad Nacional de Educación Enrique Guzmán y Valle]. http://repositorio.une.edu.pe/ bitstream/handle/UNE/2491/TD CE 1943 S1 Salazar Llerena.pdf? sequence $=1$ \&isAllowed $=y$

Borgobello, A., y Roselli, N. D. (2016). Rendimiento académico e interacción sociocognitiva de estudiantes en un entorno virtual. Educacao e Pesquisa, 42(2), 359-374. https://doi. org/10.1590/S1517-9702201606143478

Cahuana-Cuentas, M., Arias-Gallegos, W. L., RiveraCalcina, R., y Ceballos-Canaza, K. D. (2019). Influencia de la familia sobre la resiliencia en personas con discapacidad física y sensorial de Arequipa, Perú. Revista Chilena de NeuroPsiquiatría, 57(2), 118-128. http://www.scielo. $\mathrm{cl} /$ scielo.php?script=sci_arttext\&pid=S071792272019000200118\&lang=es\%0Ahttp:// www.scielo.cl/pdf/rchnp/v57n2/0717-9227rchnp-57-02-0118.pdf

Díaz-A., G., y Yaringaño-L., J. (2014). Clima familiar y afrontamiento al estrés en pacientes oncológicos. Revista de Investigación En Psicología, 13(1), 69. https://doi.org/10.15381/ rinvp.v13i1.3737

Edel Navarro, R. (1997). Factores asociados al rendimiento académico. Revista Iberoamericana de Educacion, 1-21. https://doi.org/10.4321/ S1575-18132005000200005

Gallego-Rodríguez, A., y Martinez-Caro, E. (2003). Estilos de aprendizaje y e-learning. Hacia un mayor rendimiento académico. RED. Revista de Educación a Distancia, 7, 0. https://revistas. um.es/red/article/view/25411

Garbanzo-Vargas, G. M. (2012). Factores asociados al rendimiento académico en estudiantes universitarios, una reflexión desde la calidad de la educación superior pública. Revista Educación, 31(1), 43. https://doi.org/10.15517/ revedu.v31i1.1252

Gómez-Sánchez, D., Oviedo-Marin, R., y MartinezLopez, E. I. (2011). Factores que influyen en el rendimiento academico del estudiante universitario. Educación y Humanidades, $V, N^{\circ}$ 2(junio 2011), 8 
González-Guerrero, K., Arias-Arias, N. C., y PadillaBeltrán, J. E. (2010). Incidencia del estilo de aprendizaje rendimiento académico en un curso virtual. Incidencia Del Estilo de Aprendizaje En El Rendimiento Académico En Un Curso Virtual, 31, 6-24. https://revistavirtual.ucn.edu.co/ index.php/RevistaUCN/article/view/35

González-Mariño, M. A. (2019). Factores que influyen en el rendimiento académico de residentes de Obstetricia y Ginecología de una universidad en Bogotá , Colombia. Revista U.D.C.A. Actualidad \& Divulgación Científica, 2

Jara, D., Velarde, H., Gordillo, G., Guerra, G., León, I., Arroyo, C., y Figueroa, M. (2013). Factores influyentes en el rendimiento académico de estudiantes del primer año de medicina. Anales de La Facultad de Medicina, 69(3), 193. https:// doi.org/10.15381/anales.v69i3.1140

Jiménez-Tallón, A., Ferro, J., Gómez, R., y Parra, P. (1999). Evaluación del clima familiar en una muestra de adolescentes. Revista de Psicología General y Aplicada: Revista de La Federación Española de Asociaciones de Psicología, 52(4), 453-462

Maquillón-Sánchez, J. J., y Hernández-Pina, F. (2011). Influencia de la motivación en el rendimiento académico de los estudiantes de formación profesional. Reifop, 14(1), 81-100

Mestre Escriva, V., García Samper, P., y PérezDelgado, E. (2001). Clima Familiar y Desarrollo del Autoconcepto. Revista Latinoamericana de Psicología, 33 (3), 243-259. https://www.redalyc. org/articulo.oa?id=80533301

Navarro, Ruén E. (2005). El rendimiento académico: Concepto, Investigación y desarrollo. Packaging Magazine, 8(4), 6. http://www.ice.deusto.es/ rinace/reice/vol1n2/Edel.pdf EL

Páez-Cala, M. L., y Castaño-Castrillón, J. J. (2019). Inteligencia emocional y rendimiento académico en estudiantes universitarios. Investigación En Educación Médica, 32(31), 82-91. https://doi. org/10.22201/facmed.20075057e.2019.31.18130

Patiño-Rosales, K. B. (2019). Influencia del clima familiar en el rendimiento académico de los estudiantes de la escuela profesional de odontología de la universidad peruana "Loa Andes", Huancayo - 2018 [Universidad Nacional Hermilio Valdizan]. http://repositorio.unheval. edu.pe/handle/UNHEVAL/5063

Qutishat, M., Rathinasamy-Lazarus, E., Razmy, A. M., y Packianathan, S. (2020). University students' nomophobia prevalence, sociodemographic factors and relationship with academic performance at a University in Oman. International Journal of Africa Nursing Sciences, 13, 100206. https://doi.org/10.1016/j. ijans.2020.100206

Recio-Saucedo, M. A. (2005). Enfoques de aprendizaje, rendimiento académico y satisfacción de los alumnos en formación en entornos virtuales. Pixel-Bit. Revista de Medios y Educación, 1(25), 93-115. https://www.redalyc. org/pdf/368/36802510.pdf\%0Ahttp://www. redalyc.org/articulo.oa? $\mathrm{id}=36802510$

Sandoval-Oviedo, M. E. (2017). Clima social familiar en alumnos de 4to y 5 to de secundaria de la I.E. $N^{\circ} 60188$ Simón Bolivar, noviembre 2016 [Universidad Científica del Perú]. http:// repositorio.ucp.edu.pe/handle/UCP/379

Sotelo-Castillo, M. A., Ramos-Estrada, D. Y., y Vales-García, J. J. (2011). Clima familiar y su relación con el rendiminto académico en estudiantes universitarios. XI Congreso Nacional de Investigación Educativa, 1-8. http://www. comie.org.mx/

Teresa-Vega, T., y De Dios -De Dios, Y. (2006). Beneficios Psicosociales de los grupos de apoyo: su influencia en los estilos de socialización familiar. Intervención Psicosocial, 15, 233-244

Torres-Velázquez, L. E., y Rodríguez-Soriano, N. Y. (2006). Rendimiento académico y contexto familiar en estudiantes universitarios. Enseñanza e Investigación En Psicología, 11(2), 255-270. http://www.redalyc.org/html/292/29211204/

Trevor-Mthimunye, K. D., y Megan-Daniels, F. (2019). The development and validation of an intervention for the improvement of academic performance and success of nursing students at a university in the Western Cape, South Africa. International Journal of Africa Nursing 
Sciences, 11(December 2018), 100156. https:// doi.org/10.1016/j.ijans.2019.100156

Vedaa, O., Erevik, E. K., Hysing, M., Hayley, A. C., y Sivertsen, B. (2019). Insomnia, sleep duration and academic performance: a national survey of Norwegian college and university students. Sleep Medicine: X, 1, 100005. https://doi. org/10.1016/j.sleepx.2019.100005

Verdugo-Lucero, J. C., Arguelles-Barajas, J., Guzmán-Muñiz, J., Márquez-González, C., Montes-Delgado, R., y Uribe-Alvarado, I. (2014). Influencia del clima familiar en el proceso de adaptación social del adolescentes. Psicología Desde El Caribe, 31(2), 207-222. https://doi. org/10.14482/psdc.31.2.6127

Zimmer- Ferreira, E., Neto de-Oliveira, A. M., Possani-Medeiros, S., Calcagno-Gomes, G., Cezar-Vaz, M. R., y Amorim de-Ávila, J. (2020). A influência da internet na saúde biopsicossocial do adolescente: revisão integrativa. Revista Brasileira de Enfermagem, 73(2), 1-9. https:// doi.org/https://doi.org/10.1590/0034-71672018-0766 